\title{
Raquitismo, Patogenia y Clasificación
}

\author{
DRES.: JAVIER TORRES - GOTTIA T. *, ELIANA FERNANDEZ *, EMA VALLEJOS* y MARIA EUGENIA \\ RADRIGAN *.
}

El raquitismo carencial y las formas clínicas llamadas "resistentes" a la vitamina D, habitualmente se estudian por separado; como trastorno nutritivo el primero y como alteración genética o enfermedad renal, las otras. A nuestro juicio las distintas formas de raquitismo no sólo tienen síntomas clínicos y radiológicos iguales sino además, patogenia común aunque su etiología sea diferente. Con este criterio creemos que el raquitismo se puede definir como: Trastorno del proceso normal de osificación del hueso en periodo de crecimiento activo por defecto en su mineralización. Definición que tiene la ventaja de no prejuzgar sobre su etiología y scñalar con precisión el defecto de mineralización del hueso, como hecho básico de la enfermedad.

La definición propuesta permite, además, relacionar mejor el raquitismo con la osteomalasia, que es el mismo trastorno con la diferencia de que afecta al adulto cuyo crecimiento en longitud ha terminado. Como el proceso de osificación no termina con el crecimiento, su alteración puede presentarse a cualquier edad.

El propósito de esta actualización es resumir los nuevos conceptos sobre osificación en niños normales y desnutridos, destacar la importancia del metabolismo óseo y fosfocálcico, actualizar el metabolismo y funciones de la vitamina D y relacionar estos conocimientos con la patogenia del raquitismo para proponer una clasificación de esta enfermedad que sirva de orientación al pediatra general.

\section{OSIFICACION}

Osificación u osteogénesis, son términos sinónimos que se refieren a la formación de

\footnotetext{
* Unidad de Nutrición. Departamento de Pediatría. Hosp. Roberto del Río.
}

todo el hueso, la cual comprende dos etapas distintas que son: 1) Formación de la matriz ósea y 2) Mineralización (1).

1) FORMACION DE LA MATRIZ OSEA.- La osificación se inicia con la aparición de los osteoblastos o células formadoras de tejido óseo. Estas células de intensa actividad metabólica sintetizan y segregan una substancia orgánica intercelular que es la matriz ósea, la cual está específicamente destinada a captar sales minerales de fósforo y calcio para transformarse en tejido óseo (2).

La síntesis de matriz ósea está regulada por una serie de acciones hormonales y por la disponibilidad de aminoácidos y de otros nutrientes que circulan por el plasma. Durante la evolución de la desnutrición es una de las primeras actividades anabólicas que disminuye (1) (3). Esta disminución de la secreción de matriz ósea es la responsable del retardo de osificación y del menor crecimiento en longitud que se observa en los niños desnutridos (4).

La matriz ósea representa la substancia intercelular de las otras variedades de tejido conectivo, del cual el tejido óseo es un tipo particular. Está compuesta, como todas las substancias intercelulares de fibras de colágeno, mucopolisacáridos, algunos lípidos y otras proteinas (5). Lo que la diferencia y le da su característica particular de matriz ósea es la abundancia de fibras de colágeno que constituyen el 90 a $95 \%$ de su composición mientras que los mucopolisacáridos y los otros constituyentes forman sólo el $5 \%$ restante (6).

El colágeno, que forma las fibras mencionadas, es una proteina entre cuyos aminoácidos mencionaremos únicamente la hidroxiprolina por la utilidad que presta su determinación en el estudio de la velocidad 
de crecimiento. En el metabolismo ósco una buena parte de la hidroxiprolina se elimina por el riñón sin desdoblar, lo cual permite utilizar su medición como índice de la actividad de osificación $(7) \mathrm{e}$, indirectamente del crecimiento (8). La hidroxiprolina tiene además, otra importancia: se sintetiza en el osteoblasto a partir de la prolina bajo la acción de varias enzimas y de la vitamina $C$, de modo que su síntesis está afectada en el escorbuto, lo que explica las alteraciones de la matriz ósea en esta enfermedad (1).

2) MINERALIZACION.- Es la segunda etapa del proceso de osificación. Consiste en la precipitación de sales de fosfato de calcio $\mathrm{y}$ otros jones sobre la matriz ósea en forma de cristales de hidroxiapatita.

Anteriormente se aceptaba que las sales de calcio y fósforo precipitaban sobre la matriz, exclusivamente por sobresaturación en el plasma o el líquido intersticial circundante al hueso. El calcio era el ión considerado de mayor importancia y de ahí el nombre de calcificación dado a este proceso. Actualmente el estudio de la estructura molecular del colágeno, la forma en la que se lleva a cabo la osificación y el rol preponderante del ión fosfato han permitido el desarrollo de nuevas hipótesis. En base a éstas utilizamos en este trabajo el término de "mineralización" para diferenciar específicamente el proceso exclusivo del hueso y seguimos empleando "calcificación", en forma genérica, para referirnos a las precipitaciones de sales cálcicas que pueden ocurrir normal o patológicamente sobre distintas variedades de tejido conectivo.

Hodge y Petruska han demostrado que las moléculas de colágeno que forman las microfibrillas se sobreponen linealmente, en tal forma que dejan pequeños huecos de secuencia definida, on los cuales se inicia la formación de la parte mineral del hueso (9). Sobre esta base Glimcher y Krane han elaborado su tesis que sostiene que el colágeno juega un rol central en el proceso de mineralización y afirman que el colágeno óseo, por sí mismo o por acción de determinadas enzimas incorpora el fosfato inorgánico a su molécula. La unión covalente del fósforo con la proteina permite una ordenada orientación de los iones fosfato, que son los que atraen a los otros minerales, para conformar múcleos de cristalización que se sitúan específicamente en los espacios descritos por Hodge y Petruska. Sólo sobre estos núcleos precipitan con facilidad las sales de calcio y fósforo disueltas en el plasma en proporción adecuada, de otro modo no lo hacen espontáneamente (9) (10).

Una serie de observaciones respaldan la tesis de Glimcher y Krane. Lyman A. Page (10) cita entre otras, las siguientes: 1) La ordenada organización de los cristales en tejidos en proceso de mineralización coincide con la estructura de las microfibrillas de colágeno; 2) La estructura de los cristales de hidroxiapatita depende de la organización de los iones de fosfato; 3 ) En experimentos in vitro, soluciones de calcio y fósforo que sc mantienen como tales aún a concentraciones ligeramente superiores a las fisiológicas precipitan al ponerse en contacto con el colágeno, siempre que éste conserve su estructura y composición química; 4) La administración de fosfato in vivo puede inducir la precipitación de sales de fosfato de calcio sobre el hueso, mientras que la administración de calcio solo, es menos efectiva.

Además del colágeno y el fósforo hay otros factores que influyen en la normal mineralización del hueso. Por su importancia cn la patogenia de algunas formas de raquitismo citaremos los pirofosfatos, la fosfatasa alcalina y el pH.

Los PIROFOSFATOS, son polipéptidos que se forman como derivados del metabolismo celular y de la transformación metabólica del ATP. Circulan por la sangre y se eliminan por el riñón. Normalmente inhiben la precipitación de sales cálcicas sobre todas las variedades de tejido conectivo. Su concentración en la orina impide la precipitación de sales de fosfato de calcio en el riñón, aún con niveles elevados de calciuria $\mathrm{y}$ fosfaturia (9).

La FOSFATA ALCALINA se segrega por los osteoblastos junto con la matriz ósea (1). Desdobla las moléculas orgánicas fosforadas y parece contribuir de este modo al aumento local de fósforo. Desdobla también los pirofosfatos y cumple de este modo la importante misión de neutralizar el efecto inhibidor de estos polipéptidos a nivel óseo. Se cree, además, que estimula la mineralización de la matriz ósea por mecanismos todavía no bien precisados que parecen actuar sobre la misma matriz (11).

Un pH bajo dificulta la mineralización y favorece la disolución de los cristales de hidroxiapatita. Lo inverso ocurre con $\mathrm{pH}$ alto $(11)$.

En síntesis, el proceso de mineralización todavía no está enteramente conocido pero 
depende de factores locales y humorales. Se puede afirmar que los hechos más importantes de esta segunda etapa de la osificación, son : 1) La incorporación de fósforo a la molécula proteica del colágeno, adecuadamente estructurado en el interior de las microfibrillas, para formar los núcleos de cristalización. 2) La expansión de la cristalización sobre estos núcleos, gracias a la existencia de niveles adecuados de calcio y fósforo en el plasma. 3) Que este proceso puede ser inhibido o estimulado por varios factores entre los que están los pirofosfatos y el $\mathrm{pH}$ ácido como inhibidores y la fosfatasa alcalina y el ión fosfato como estimulantes.

\section{OSTEOPOROSIS Y RAQUITISMO}

EI proceso de osificación con sus dos etapas, secreción de matriz y mineralización, constituyen en conjunto sólo la primera parte del metabolismo óseo: la anabólica, a la cual sigue la parte catabólica o de reabsorción del hueso. Estos dos procesos se mantienen activos y sin interrupción a lo largo de toda la vida. Se calcula que un adulto joven renueva cada año el 50\% de la estructura total de determinados huesos (9). La osteogénesis y la osteolisis están equilibradas recíprocamente por la actividad de las células del tejido óseo. Se sostiene ahora que la célula osteoprogenitora del mesénquima tiene una sola línea de diferenciación cuya secuencia de transformación es la siguiente: Célula precursora - preosteoclasto - osteoclasto - preosteoblasto - osteoblasto - osteocito (12). Dentro de esta serie de maduración sucesiva el osteoclasto es responsable de la reabsorción ósea, el osteoblasto de la formación y el osteocito puede cumplir ambas funciones. Con este criterio es más fácil comprender la aparente paradoja de que al estimularse la reabsorción ósea por cualquier mecanismo iónico u hormonal, se estimule también su formación y que igual cosa ocurra con los mecanismos de inhibición que al inhibir la reabsorción ósea inhiben también la osteogénesis.

El balance entre osificación y reabsorción normalmente es positivo a favor de mayor osificación en el niño que crece, es equilibrado en el adulto y tiende a hacerse negativo en la senectud por predominio de la reabsorción sobre la osificación (11). En términos generales se puede decir que existe osteoporosis cuando el balance entre osificación y reabsorción es negativo para el hueso, ya sea por exceso de reabsorción o por menor secreción de matriz para la osificación (13 (14).

El déficit crónico de calcio en el organismo provoca balance negativo con osteoporosis por exceso de reabsorción. La desmineralización implica reabsorción de la matriz ósea en primer término; la remoción de los minerales es posterior (19).

En los niños desnutridos, la menor capacidad de sintesis proteica conduce a menor osificación con menor formación de matriz ósea (13) (15). Esto ocasiona menor crecimiento y formación de huesos rarefactos muy semejantes a los de la osteoporosis senil (3) (16). Como la desnutrición, particularmente en los casos graves, se acompaña de inactividad, puede producirse también la llamada "osteoporosis por desuso" (17) (15). No se ha aclarado si ésta se debe a mayor reabsorción ósea. Se ha demostrado en cambio, que la osificación y la reabsorción óseas, se regulan por mecanismos preso-eléctricos que se producen en la dinámica del movimiento activo que es uno de los condicionantes del remodelamiento continuo que tiene el hueso. La energía eléctrica creada por presión se convierte en estímulo químico, que provoca mayor actividad de osificación, mayor secreción de matriz ósea y mayor mineralización (11) (9). Durante el reposo prolongado, disminuye el estímulo para la osificación, lo que podría explicar la osteoporosis por desuso.

El raquitismo es una alteración distinta que afecta, como ya hemos dicho, específica y exclusivamente a la calidad del hueso y a otro equilibrio, el que existe entre secreción de matriz y su mineralización. Cuando la mineralización se retrasa, o es insuficiente en proporción al ritmo de secreción de matriz ósea, se origina raquitismo u osteomalasia.

La acumulación de matriz ósea sin mineralizar, forma un tejido blando que se conoce con el nombre de "osteoide". Este tejido da la blandura patológica al hueso en su conjunto, deforma las zonas de crecimiento de los huesos largos (unión diáfiso-epifisaria), la unión de las costillas con los cartílagos costales y provoca todas las alteraciones anatómicas responsables de los síntomas clínicos y radiológicos de la esteomalasia y del raquitismo bien conocidos (18).

Se comprende fácilmente que el equilibrio entre secreción de matriz y mineralización, es más susceptible de alterarse por defecto de esta última etapa en los períodos de mayor osificación, en los cuales hay mayor secreción de matriz ósea. Por eso el ra- 
quitismo se presenta más frecuentemente en niños con normal velocidad de crecimiento y más todavía en los que esta velocidad está aumentada como en los prematuros o desnutridos on fase de recuperación. Es menos frecuente an 'os desnutridos agudos, con menor velocidad de desarrollo.

I as dos alteraciones: osteoporosis -pérdida de snbitancia ósea- y raquitismo mala mincralizarión-, son diferentes y obedecen a cousas distintas. Tanaka y DeLuca insisten en la importancia de la hipofosfemia más que de la hipocalcemia en el raquitismo. La hipocalcemia sin hipofosfemia conduce a osteoporosis (19). Sin embargo, la asociación de osteoporosis y raquitismo es frecuente por la particularidad del hueso de que cualquier aumento de la reabsorción ósea se acompaña de aumento también, aunque en menor grado, de secreción de matriz. Esta matriz debe a su vez mineralizarse en las condiciones desventajosas de hiperparatiroidismo secundario a la hipocalcemia lo cual pucde ocasionar raquitismo. Por otra parte el raquitismo carencial, que supone menor absorción intestinal de calcio por falta de vitamina $D$, es natural que se acompañe de mayor reabsorción ósea por hipocalcemia y se asocie a cierto grado de osteoporosis.

\section{METABOLISMO FOSFOCALCICO}

El metabolismo fosfocálcico está en íntima relación con el proceso de remodelamiento continuo del hueso. Pero es necesario tener en cuenta que cl fósforo y más el calcio cumplen funciones cuya prioridad no es cl mantenimiento de la osificación, sino que, por el contrario, el hueso es el principal depósito de reserva de iones minerales, para el mantenimiento de la homeoestasis. Así como el hueso es el primero en sacrificar la síntesis de matriz orgánica para compensar deficiencias nutritivas, sus iones minerales: $\mathrm{Ca}, \mathrm{P}, \mathrm{Na}, \mathrm{Mg}, \mathrm{CO} 3$ y otros, están también continuamente a disposición de los requerimientos plasmáticos y pueden abandonar el hueso para corregir desequilibrios iónicos o ácido básicos aún a cambio de desmineralizar el hueso (20).

El calcio precisa una absorción intestinal activa que varía en función de las necesidades orgánicas, gracias a la actividad de la vitamina D. Su concentración total en el plasma es de $10 \mathrm{mg}$. por $100 \mathrm{ml}$. El $46 \%$ circula ligado a la proteina, el $47,5 \%$ al estado iónico y el $6,5 \%$ en forma de citratos, fosfatos y otras sales (21).
Las recomendaciones nutricionales son de $500 \mathrm{mg}$. diarios para lactantes y alrededor de $800 \mathrm{mg}$. para niños. En la mujer embarazada suben a 1.700 y en la lactancia a $1.800 \mathrm{mg}$. por día (22). La eliminación se la hace en su mayor parte $(7 / 8)$ por el intestino, pero la octava parte que se elimina por el riñón es la variable que regula la eliminación. La calciuria normal es de alrededor de $125 \mathrm{mg}$. por día para el adulto (11). Royer recomienda que en el niño se exprese en $\mathrm{mg} / \mathrm{kg} / 24$ hs. y afirma que su valor es generalmente inferior a $3 \mathrm{mg}$. Una calciuria superior a $6 \mathrm{mg} / \mathrm{kg} / 24 \mathrm{hs}$. debe considerarse hipercalciuria eventualmente patológica (20).

De la calcemia normal dependen la exitabilidad neuromuscular, la función de las membranas celulares, una variedad de funciones enzimáticas y endocrinas (22). Particularmente en su estado iónico no puede variar sino en muy estrechos limites, ya que variaciones ligeras conducen a trastornos graves, algunos agudos como la tetania.

Se sabía que el fósforo tiene una absorción intestinal pasiva, indirectamente beneficiada por una mejor absorción del calcio, pero parece que además, su absorción es directamente estimulada por la vitamina $D$ (23) (24). Las recomendaciones diarias son de alrededor de $220 \mathrm{mg}$. (26). Su concentración en el plasma es variable, cambia con la edad, la dieta y se regula por su eliminación renal, ya que prácticamente es la única vía de climinación. La fosfemia normal en el nino es de 5 a $6 \mathrm{mg}$. por $100 \mathrm{ml}$. En el adulto es sólo de 2,5 a 4,3 mg. El 12\% está ligado a la proteina, el 53\% circula como ión fosfato y el resto combinado con sales de $\mathrm{Na}$, Ca y Mg. (21). La fosfaturia varía entre 150 y $1.500 \mathrm{mg}$. por 24 hs. Los niños y más aún los recién nacidos eliminan cantidades relativamente escasas (27). Más útil es recordar que normalmente el $66 \%$ del fósforo ingerido se elimina por el riñón (11).

El fósforo cumple funciones diferentes al calcio en el plasma. Participa en la formación de moléculas importantes (ácido ribonucleico, fosfolípidos, etc.). Lo que interesa para nuestro estudio es que a través de su participación en la formación del ATP contribuye a mantener el tono muscular, cuya disminución es uno de los síntomas del raquitismo, y que la mayor concentración de fosfato determina incremento de la transformación celular de los osteoclastos en osteoblastos y mayor actividad de éstos (22). Como además, los núcleos de cristalización que inician la mineralización del hueso, se 


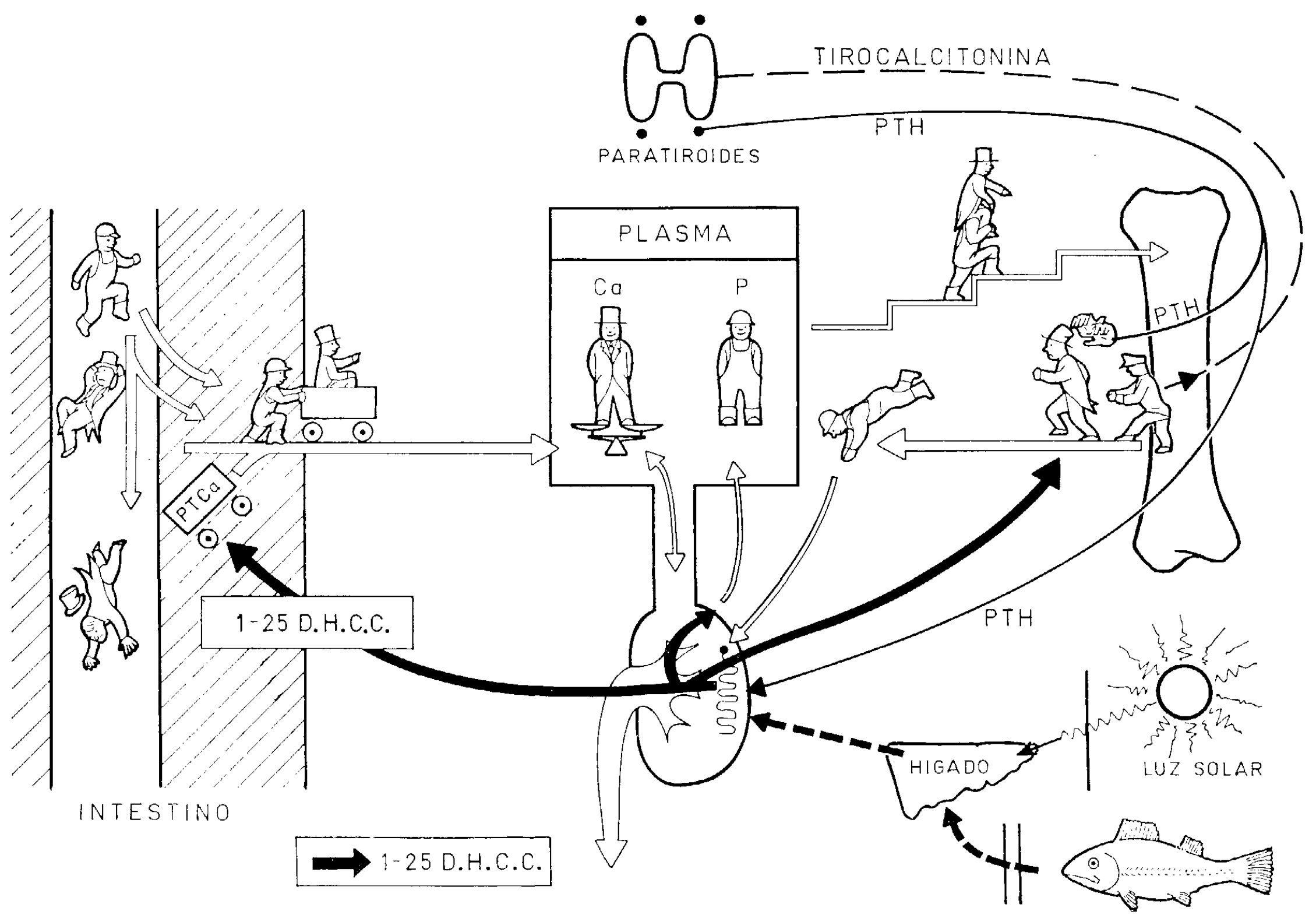

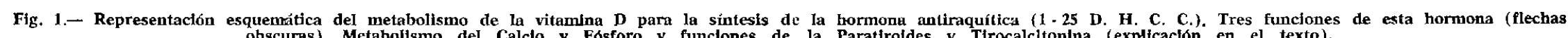
obscuras). Metabolismo del Calclo y Fósforo y funclones de la Paratiroldes y Tirocalcitonina (explicación en el texto). 
forman por incorporación de moléculas de fósforo al colágeno (9), podemos afirmar que el fosfato es importante en las dos etapas de la osificación: secreción de matriz y mineralización. Casi todas las formas de raquitismo se acompañan de hipofosfemia (28) (19). Se puede decir que el fósforo es el ión que arrastra al calcio fucra del plasma para mineralizar el hueso, actuando así como hipocalcemiante por un lado y como estimulante de la mineralización por otro (Fig. 1).

La fosfemia puede variar en ciertos límites mientras que la calcemia se mantiene casi sin variación por efecto de varias hormonas particularmente por acción de la Parathormona y la Tirocalcitonina.

Las funciones de la Parathormona pueden resumirse en tres, todas destinadas a mantener la calcemia: 1) Contribuye a la absorción intestinal del calcio estimulando la síntesis del principio hormonal de la vitamina $\mathrm{D}$ en el riñón. No tiene acción propia sobre el intestino (29). 2) Promueve la reabsorción ósea con retorno del calcio y fósforo al plasma, en presencia del metabolito activo de la vitamina D (29). En una primera fase la PTH aumenta el número y la actividad de los osteoclastos y reduce el número y la actividad de los osteoblastos. En una segunda fase la estimulación de las células osteoprogenitoras no sólo aumenta los osteoclastos sino también el número de células que llegarán a transformarse en osteoblastos, con lo que indirectamente estimula tambićn la formación ósea, aunque en menor proporción que la reabsorción (22). 3 ) Aumenta la fosfaturia bloqueando la reabsorción tubular del fósforo filtrado por los glomérulos, con lo que defiende indirectamente la calcemia al neutralizar el efecto hipocalcemiante del fósforo.

En síntesis la paratiroides en respuesta a una hipocalcemia incrementa las dos fuentes de calcio que tiene el plasma: la intestinal y la ósea y disminuye la fosfemia.

La Tirocalcitonina, hormona secretada por las células $\mathrm{C}$ del tiroides, es antagónica de la parathormona. En el ciclo celular, inhibe la función de los osteoclastos, aumenta su transformación en osteoblastos e inhibe la activación de las células osteoprogenitoras, por eso, si bien inicialmente reduce la reabsorción ósea y aumenta su formación, en una fase crónica, no sólo disminuye la reabsorción sino también la osificación (22) (12).
VITAMINA D, METABOLISMO Y FUNCIONES

En la historia del estudio de la vitamina $\mathrm{D}$, se pueden distinguir dos etapas: la primera desde 1920 a 1940 , en la cual se busca el "principio antiraquítico" y culmina con la identificación de la vitamina D2 o ergocalciferol, de origen vegetal y la vitamina D3 o colecalciferol, de origen animal. La segunda etapa, se inicia en 1955 con los trabajos con isótopos marcados de Kodicek (30) pero se desarrolla más desde 1969 en adelante con la identificación del 25 hidroxicolecalciferol por DeLuca y colaboradores (31) (32).

Actualmente, el estudio del metabolismo de la vitamina $D$ ha llegado a establecer que la forma activa de esta vitamina no es el ergo ni el colecalciferol sino un principio hormonal que se forma en el hígado y el riñón, a partir de la vitamina $D$ ingerida con la dieta o sintetizada en la piel. El proceso de síntesis del principio hormonal de la vitamina D puede resumirse en la siguiente forma:

1) ABSORCION.- La vitamina D3 activada por irradiación de la piel pasa por vía sanguínea al hígado (33). La vitamina $D$ ingerida por vía oral debe ser absorvida a través de la mucosa intestinal. Como vitamina liposoluble requiere para su absorción, todas las condiciones necesarias para la absorción de las grasas. Son indispensables las sales biliares, la suficiente proporción de lipasa y la integridad de las células del epitelio intestinal. Una vez absorvida por la mucosa intestinal, la vitamina $\mathrm{D}$ ingerida es llevada también al hígado por vía linfática (34) (35).

2) METABOLISMO.- El metabolismo interno de la vitamina $\mathrm{D}$ empieza en el hígado. La célula hepática por acción de la calciferol 25 hidroxilasa añade a la molécula de colecalciferol un grupo hidroxil en el carbono 25 para formar el 25 hidroxi-colecalciferol (25 HCC) o 25 hidroxi-vitamina D3$25(O H) D 3-$ como también se puede designar (32).

El hígado parece formar además otros metabolitos todavía en estudio (33). El 25 HCC y los otros metabolitos se conjugan con el ácido glicurónico y se segregan junto con la bilis al intestino de donde se reabsorven y vuelven al hígado participando de la circulación enterohepática de las sales biliares, antes de pasar al plasma sanguíneo. Por este motivo, las fístulas biliares pueden pro- 
ducir agotamiento de la vitamina $\mathrm{D}$ independientemente de los trastornos de absorción (36).

Se acepta que la vitamina D2 o ergocalciferol, sufre el mismo proceso metabólico que el colecalciferol, por acción de la misma hidroxilasa hepática (37).

La $25(\mathrm{OH})$ D3 pasa al plasma y se considera que es la forma circulante de la vitamina D (38) (39). Su producción hepática tiene un mecanismo de autoregulación por feed-back poco sensible, se regula por cambios de concentración relativamente grandes (40). Por la circulación general llega al riñón donde se cumple la segunda y última etapa metabólica.

En el riñón, otra enzima, la 25-hidroxicolecalciferol-1-hidroxilasa contenida en las mitocondrias de las células tubulares del riñón convierte el $25 \mathrm{HCC}$ en otro compuesto dihidroxilado: el 1-25 dihidroxicolecalciferol $-1-25$ DHCC - que es la forma activa de la vitamina $\mathrm{D}$ actualmente considerada una hormona ya que es producto de la síntesis y secreción celular, se vacía directamente al plasma y cumple su acción a distancia sobre órganos efectores (41).

La síntesis de 1-25 DHCC está también regulada por un mecanismo de feed-back pero de sensibilidad mucho mayor a la del hígado para el 25 HCC y está además, bajo el control de variaciones hormonales y iónicas. La parathormona estimula la formación de 1-25 DHCC y la calcitonina la inhibe, influyen también variaciones en la concentración de calcio y fósforo (42) (43). El riñón no sintetiza sino las cantidades necesarias; en condiciones normales si los niveles de calcemia se elevan se forman otros derivados metabólicos cuyas funciones están en pleno estudio. Algunos de estos metabolitos tienen acción sobre la absorción intestinal del calcio con poco o ningún efecto sobre la reabsorción ósea y otros son inactivos. Su importancia es grande para investigaciones posteriores (44) (45) (29).

FUNCIONES DE LA VITAMINA D.Cuando nos referimos a las funciones de la vitamina $\mathrm{D}$ se sobreentiende las del metabolito activo o principio hormonal 1-25 DHCC, llamado también 1-25 Dihidroxivitamina $D$ 1-25- $(\mathrm{OH})$ 2-D3 (37). Hasta el momento se han precisado dos funciones y están en estudio otras.

1.- ABSORCION INTESTINAL DE CALCIO.- A nivel del aparato digestivo la 1-25-(OH) 2-D3 induce la formación de una proteina fijadora del calcio en la célula del epitelio intestinal (46) (47) (48) (49) (50). Esta proteina es la que absorve el calcio del lumen intestinal y la traspasa al plasma sanguineo a través de la célula epitelial.

\section{2.- REABSORCION DEL CALCIO} OSEO.- A nivel óseo la 1-25-( $\mathrm{OH})$ 2-D3 contribuye a la reabsorción del calcio óseo. La reabsorción es estimulada por la parathormona, pero es la vitamina D la que posibilita el traslado del calcio desde el hueso al plasma (51) (52).

OTRAS FUNCIONES. - Las dos funciones mencionadas explican sólo en forma relativa la evidente acción antiraquítica de la vitamina D. Estudios en perros (53) han demostrado una tercera función que estimula directamente la reabsorción tubular del fósforo. Pechet (54) demuestra también que la 1-25 (OH) 2 D3 aumenta la reabsorción tubular del fósforo y que esta acción es favorecida por el ión calcio.

Se ha vuelto a sostener que la vitamina D tiene acción directa sobre la matriz ósea para estimular directamente su mineralización (55). DeLuca (29) afirma que con los nuevos metabolitos de gran actividad biológica se podrá dilucidar en breve esta posibilidad.

Aunque la 1-25 (OH) 2 D3 cumple todas las funciones actualmente conocidas no se excluye la importancia que puedan tener otros metabolitos, en funciones específicas. Por ejemplo la 24-25 (OH) 2 D3 que aparece como efecto de la autoregulación por feed-back de la 1-25 (OH) 2D (56) es inactiva, pero al añadirse en el riñón otra hidroxilación en el carbono -1- para formar la 124-25 Trihidroxivitamina D3 se hace activa para aumentar la absorción intestinal del calcio con poco o ningún efecto sobre la reabsorción ósea (45).

El elevado costo de la 1-25 (OH) 2 D3 y las dificultades para su administración, han llevado a investigar metabolitos analógos, lográndose sintetizar la -1-alfa hidroxivitamina D3 que tiene acción sobre la absorción intestinal del calcio, la mineralización del hueso y la reabsorción ósea incluso en animales nefrectomizados (29) o en pacientes con insuficiencia renal (57).

La -1-alfa (OH) D3 y la $25(\mathrm{OH})$ D3 parecen ser la clave de futuros tratamientos en alteraciones del metabolismo de la vitamina D por defectuosa hidroxilación en el Carbono 1 o en el 25 , respectivamente.

Con el mejor conocimiento del metabo- 
lismo de la vitamina $D$ se explica la acción del dihidrotaquisterol y de su forma hidroxilada en el C 25 ó $25(\mathrm{OH})$ dihidrotaquisterol que es más efectiva (58). Su rápida acción para estimular la reabsorción ósea y a dosis altas la absorción intestinal puedc deberse a su parecido químico con la 1-25(OH) 2 D3, pero hasta el momento no se ha demostrado que pueda convertirse en 1-25 (OH) 2 D3, lo que explica la aparente paradoja de ser menos efectivo que las formas tradicionales de vitamina D2 ó D3 en el raquitismo carencial o cuando no hay alteracićn del metabolismo de la vitamina D (33).

\section{CLASIFICACION DE LAS FORMAS CLINICAS DE RAQUITISMO}

La profilaxis sistemática y el enriquecimicnto de alimentos con vitamina $\mathrm{D}$, han hecho que el raquitismo por carencia primaria de esta vitamina sea cada vez menos frecuente o tenga manifestaciones clínicas leves. Pero por otra parte destacan ahora, más que antes, formas graves a las que se ha atribuido distintas etiologías y cuya aparente heterogeneidad dificulta su diagnóstico.

De la revisión realizada podemos concluir que todas las formas clínicas de raquitismo se producen por insuficiente mineralización de la matriz ósea, lo cual en último análisis resulta de una deficiencia de fósforo o de inhibición de la mineralización. Con este concepto nos parece posible englobar las distintas formas clínicas de raquitismo en una sola entidad nosológica y clasificarlas por su ctiopatogenia en la siguiente forma:

La hipofosfemia, que es el hecho común a casi todas las formas de raquitismo, es resultado de una carencia del principio activo de la vitamina $D$, la cual a través de una hipocalcemia inicial con hiperparatiroidismo secundario provoca hiperfosfaturia; o de hiperfosfaturia de otro origen sin carencia vitamínica. Entre estas últimas, que hemos denominado "no carenciales", las dos primeras: Hipofosfatemia Familiar y Raquitismo Oncogénico, están bien definidas, como veremos luego. No ocurre lo mismo con las tres formas clínicas menos bien conocidas, que con muchas interrogantes las mantenemos como no carenciales y provocadas por inhibición de la mineralización.

La investigación con los metabolitos activos de la vitamina $D$, está haciendo que formas clínicas llamadas "resistentes" y para las que se invocaban etiologías ajenas a la vitamina $D$, pasen a definirse como secundarias a defectos del metabolismo hepático o renal de esta vitamina, así, hay argumentos para sostener que el raquitismo de la insuficiencia renal crónica se debe también a trastorno del metabolismo de la vitamina D a nivel renal. Un mejor conocimiento y nuevas experiencias con la hormona antiraquítica probablemente permitirán simplificar aún más una clasificación etiopatogénica.

\section{RAQUTTISMO POR CARENCIA DE VITAMINA D.}

Partiendo de la base de que la acción fisiológica de esta vitamina la cumple la hormona $1-25$ dihidroxivitamina $\mathrm{D}_{3}$, para cuya síntesis el organismo necesita el aporte de colecalciferol o ergocalciferol, conocidos como vitamina $\mathrm{D}_{3} \circ \mathrm{D}_{2}$, las formas carenciales de raquitismo las hemos dividido en dos: Primaria y Secundaria.

CARENCIA PRIMARIA.- Consideramos como tal al déficit de $1-25(\mathrm{OH})_{2} \mathrm{D}_{3}$ por insuficiente aporte exógeno de vitamina $\mathrm{D}$. Para que se produzca raquitismo por esta causa, deben asociarse menor ingestión vitamínica, con deficiente irradiación solar ya que en condiciones climáticas adecuadas, la

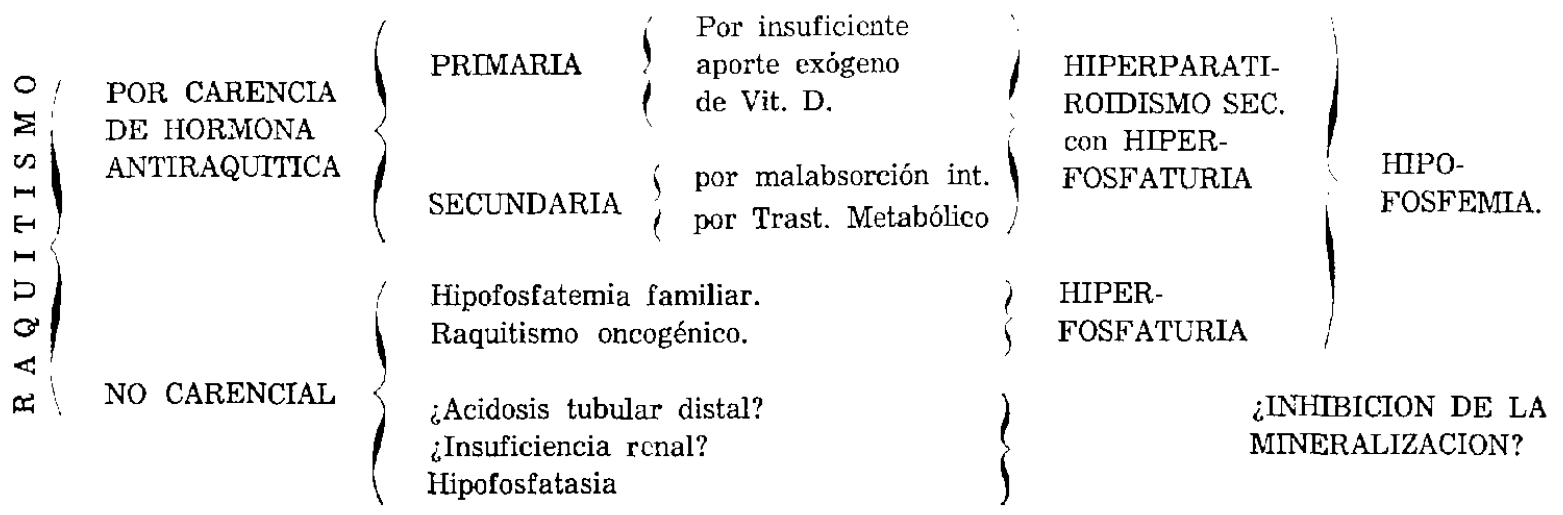


sintesis cutánea por sí sola es suficiente para cubrir los requerimientos de vitamina $\mathrm{D}$ (33) (39). Así se explica que el raquitismo por insuficiente aporte dietético sea de grado leve o no se presente sino en regiones geográficas o bajo condiciones de vida especiales.

CARENCIA SECUNDARIA-— Puede deberse a: 1) Malabsorción intestinal y 2) Trastornos metabólicos de la vitamina.

1) MALABSORCION INTESTINAL.Esta se produce en todas las afecciones que perjudican las absorción de las grasas. Puede presentarse carencia secundaria de vitamina $\mathrm{D}$ cuando disminuye la secreción de bilis al intestino, en afecciones pancreáticas que disminuyen la lipasa y en las alteraciociones de la mucosa intestinal que provocan malabsorción: enfermedad celíaca, desnutrición grave, etc. En la enfermedad celíaca, la atrofia de las vellosidades provocada por el gluten no sólo afecta la absorción de la vitamina $D$, sino que altera la capacidad de síntesis proteica de la célula. La vitamina D parenteral o la irradiación solar suficiente compensan la carencia vitamínica secundaria pero no corrigen la menor absorción intestinal de calcio que resulta de la incapacidad de la célula epitelial para formar la proteína fijadora del calcio (33) (59).

2) TRASTORNOS METABOLICOS.- EI metabolismo que sufre la vitamina $D$ y el mayor o menor grado de eficiencia con que se cumple, explican las variaciones de los requerimientos individuales. Además de estas variaciones fisiológicas, pueden presentarse trastornos en su metabolismo, ya sea a nivel hepático o renal.

2a) TRASTORNOS METABOLICOS HEPATICOS. - Se ha demostrado que la síntesis de 25 hidroxivitamina $D$ puede alterarse por trastornos del mecanismo enzimático de los microsomas de la célula hepática con formación de metabolitos inactivos, incapaces de transformarse después en 1-25 dihidroxi-vitamina D (56) 60). Se han descrito casos de raquitismo secundario a hepatitis (61) que curan con la administración de 25 hidroxivitamina D. Está también demostrado que el fenobarbital, la fenitoína y otros productos antiepiléticos interfieren con esta primera etapa metabólica de la vitamina D lo que conduce a raquitismo $u$ osteomalasia (60) (62) (63) (64) (65). Se afirma que 25 a $30 \%$ de la población tratada con anticonvulsivantes tiene niveles subnormales de calcio y aumento de fosfatasa alcalina (66). Pero el raquitismo se presenta solo entre 3,5 y $7 \%$ de pacientes hospitalizados con tratamiento por más de un año y particularmente cuando tienen infecciones recurrentes y menor exposición al sol (67). Parecería que la alteración en la síntesis de 25 $(\mathrm{OH}) \mathrm{D}$ en el hígado por los anticonvulsivantes no es total ya que se previene aumentando la dosis de vitamina $\mathrm{D}$ a 4.000 unidades diarias (68), apenas 10 veces más de las recomendaciones habituales. Usando directamente el metabolito hepático, la dosis de tratamiento sería de 50 unidades de 25 $(\mathrm{OH}) \mathrm{D}$ por día (67).

2b) TRASTORNOS METABOLICOS RENALES. - La síntesis de 1-25 dihidroxi-vitamina $\mathrm{D}$ puede alterarse directamente en una serie de afecciones que comprometen el túbulo proximal renal que es donde se sintetiza este metabolito activo $u$ hormona antiraquítica.

Sindrome de Fanconi.- Prácticamente todas las tubulopatías renales son susceptibles de alterar la síntesis de hormona antiraquítica y provocar raquitismo. El túbulo proximal reabsorve la glucosa, los aminoácidos, el fósforo, y el 85 a $90 \%$ del bicarbonato del filtrado glomerular. Cuando estas funciones se comprometen en conjunto por cualquier causa, se considera que existe un sindrome de Fanconi (69) el cual, puede ser hereditario o secundario a cistinosis, intoxicación con metales pesados o tetraciclinas vencidas.

Antes de conocer la formación de la hormona antiraquítica, era lógico suponer que el raquitismo asociado al sindrome de Fanconi era producido por algunas de las anomalías del túbulo, particularmente la fosfaturia. Se pensaba así que esta forma de raquitismo era independiente de la función normal de la vitamina $D$. El hecho de que en el túbulo proximal del riñón coincidan el órgano secretor de la hormona antiraquítica $\mathrm{y}$ el efector de una de sus funciones: la reabsorción del fósforo, (24) (25) (70) dificulta precisar cuales de los raquitismos obedecen a déficit de síntesis de 1-25 $(\mathrm{OH})_{2}$ $\mathrm{D}$, corregible por la administración de esta hormona y cuales otros son provocados por defecto renal, no corregible por ella (71).

Por otra parte, se ha observado (72) (73) que el hiperparatiroidismo, común a todas las formas de raquitismo carencial, provoca una alteración funcional del segmento proximal del túbulo renal que produce los sínto- 
mas que caracterizan al sindrome de Fanconi, que en estos casos resulta más bien consecuencia y no causa del raquitismo.

En el Hospital Roberto del Río, hemos observado una niña de 2 años de edad con raquitismo asociado a sindrome de Fanconi cuya causa no pudo ser precisada (74).

Raquitismo Vitamino-dependiente o Pseudo-carencial.- Aunque esta designación puede generalizarse a todas las formas que describimos como carencias secundarias, más específicamente corresponde al raquitismo refractario hereditario de tipo autosómico recesivo (56) (37) (75) (76) que ahora se ha logrado demostrar se debe a deficiencia genética de la enzima 25 hidroxicalciferol -1- hidroxilasa en las células tubulares del riñón (77).

La identificación de esta forma clínica y su separación del heterogéneo grupo de los mal llamados raquitismos vitamino resistentes, es uno de los avances más positivos. Su estudio ha permitido a Fraser establecer la dosis, que podría ser fisiológica, de 1 - 25 $(\mathrm{OH})_{2}$ D y equipararla con los requerimientos tanto de vitamina $\mathrm{D}$, como de $25(\mathrm{OH})$ D. No se conoce la proporción exacta de conversión de vitamina $\mathrm{D}$, en 25 ( $\mathrm{HO}$ ) D y en 1 - $25(\mathrm{OH})_{2} \mathrm{D}$, pero en la rata y probablemente en el hombre es de: 10:3:1 (37), de modo que las 400 unidades de vitamina $\mathrm{D}$ que se recomiendan como aporte diario, que en peso corresponden a 10 microgramos, serían equivalentes a 3 microgramos de 25 $(\mathrm{OH}) \mathrm{D}$ y a 1 microgramo de $1-25(\mathrm{OH})_{2}$ D. Los raquitismos vitamino-dependientes, curan con esta dosis fisiológica de un microgramo de hormona antirraquítica, mientras que se necesitan dosis de 1.660 microgramos de vitamina D ó 500 microgramos de $25(\mathrm{OH})$ D. La proporción de 10:3:1 considerada normal, cambia así a 1.700: 500:1 en esta forma de raquitismo. Proporción que hay que tomar en cuenta para el tratamiento con vitamina $D$ si no se dispone de metabolitos activos. El dihidrotaquisterol y el 1 - alfa - hidroxicolecalciferol, pueden también ser útiles a dosis menores: 25 microgramos de 1 - alfahidroxicolecalciferol (57). La dosis señalada de 1.660 microgramos corresponde a 66.400 unidades de vitamina $D$. Es natural que esta dosis varíe con el grado de insuficiencia enzimática. Un caso tratado por nosotros no respondió a la dosis de 100.000 unidades, pero sí lo hizo a 200.000 unidades diarias de vitamina $\mathrm{D}$ por vía oral (74).
PATOGENIA DEL RAQUITISMO POR CARENCIA DE HORMONA ANTIRRAQUITICA.- Todas las formas clínicas de raquitismo carencial, sean primarias o secundarias, se producen por carencia de $1-25(\mathrm{OH})_{2}$ D. Esta carencia provoca malbsorción de calcio y probablemente también de fósforo. En una primera etapa se produce hipocalcemia con fosfemia todavía normal. En pocos días se pasa a la segunda etapa en la cual en respucsta a la hipocalcemia aumenta la parathormona circulante, que normaliza la calcemia pero aumenta la fosfaturia y aminoaciduria; aparece hipofosfemia. En la tercera etapa, vuelve la hipocalcemia y se mantiene la hipofosfemia con hiperfosfaturia y aminoaciduria. Esta evolución explicada por la inter-relación de la parathormona y la vitamina $\mathrm{D}$, destaca la importancia del hiperparatiroidismo secundario (76) en todas las formas carenciales del raquitismo.

Las expresiones clínicas diferentes de un mismo trastorno, probablemente dependen de las variaciones en la magnitud del daño y de la edad en que éste se inicia.

\section{RAQUITISMO NO CARENCIAL}

Corresponden a este grupo las afecciones que provocan raquitismo por exagerada eliminación renal de fósforo u otra alteración pero sin compromiso de la síntesis y secreción de la hormona antiraquítica. Estas afecciones, lógicamente, no se benefician con la administración de $1-25(\mathrm{OH})_{2}$ D u otros metabolitos activos.

En el momento actual hay 2 formas bien definidas y 3 que requieren mayor estudio.

Hipofosfatemia Familiar.- Es la forma clínica mejor precisada dentro de las no carenciales. Corresponde a las formas de raquitismo de forma hereditaria dominante ligada al cromosoma $X$ (78) que ahora se sabe es un trastorno del metabolismo del fósforo con alteración de su transporte transepitelial. El raquitismo resulta de la hiperfosfaturia por mala reabsorción tubular del fósforo filtrado por el glomérulo y probablemente por disminución de la absorción intestinal de fósforo en algunos pacientes (76) (79) (80). Durante mucho tiempo se la estudió como diabetes fosfatúrica confundida con las formas actualmente conocida como pseudocarenciales o vitamino D dependientes. La diferenciación es importante ya que en la hipofosfatemia familiar ligada al cromosoma $\mathrm{X}$ no está alterada la síntesis de hormona antiraquítica, no res- 


\section{FORMAS CLINICAS DE RAQUITISMO ETIOPATOGENIA}

CARENCIAL

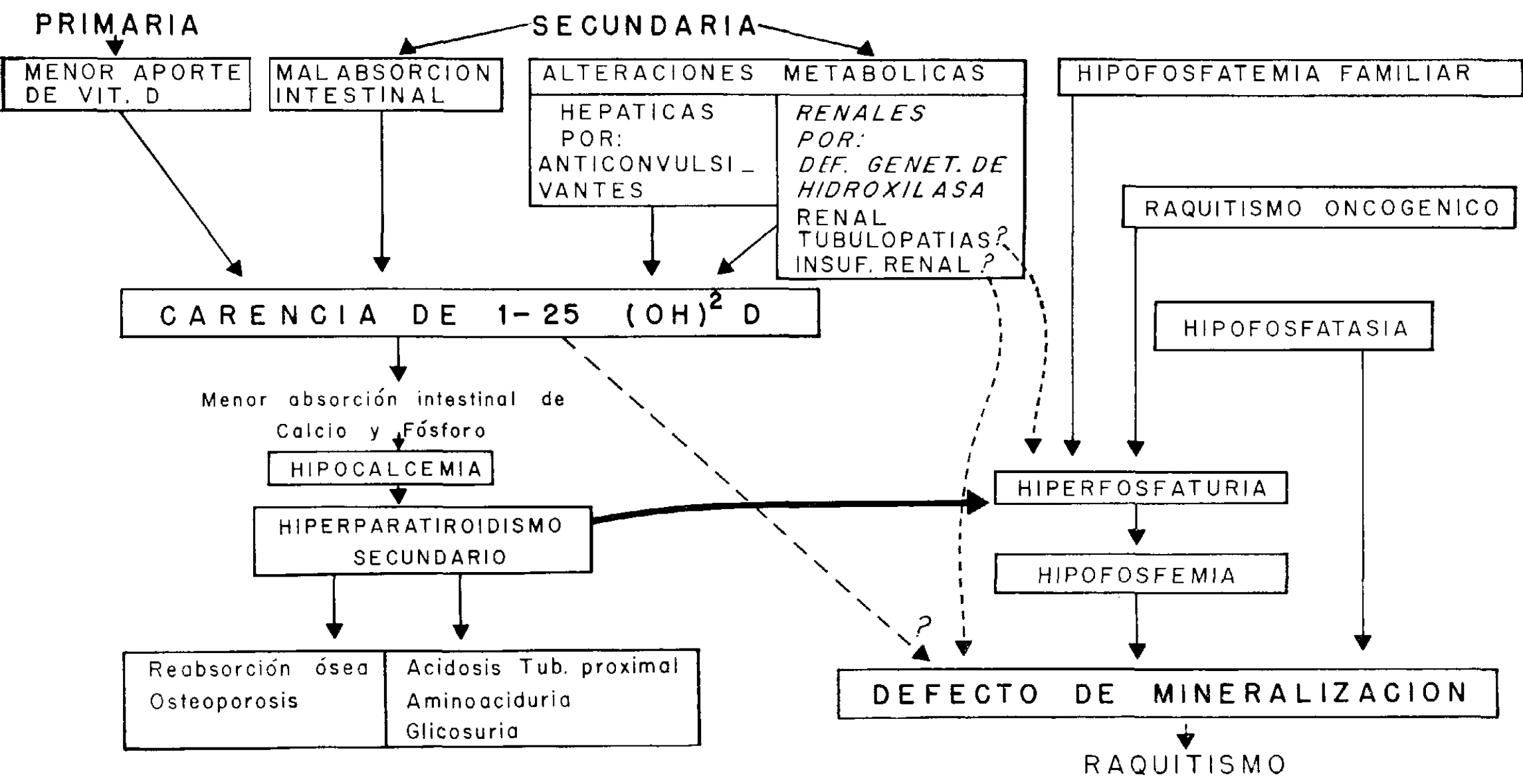


ponde al tratamiento con $1-25(\mathrm{OH})_{2} \mathrm{D}$ pero cura bien con la administración de fósforo (79) (74).

Raquitismo Oncogénico.- Es el término propuesto para designar formas adquiridas de raquitismo sccundario a determinados tumores que provocan hipofosfatemia. Curan al extirparse el tumor, corrigiéndose la hipofosfatemia y la osteomalasia o raquitismo mientras que la terapéutica con $1-25(\mathrm{OH})_{2}$ $\mathrm{D}_{3}$ no da ningún resultado (81).

Acidosis Tubular Distal.- El síndrome descrito por Albright en 1940 se produce por insuficiencia del segmento distal del túbulo renal (82). Parecería provocar raquitismo sin carencia de vitamina $D$, ya que es un hecho conocido que al curar la acidosis cura el raquitismo (83). Probablemente la mayor solubilidad de los fosfatos ácidos de calcio dificulta la precipitación de estas sales y la mineralización de la matriz ósea (9), además de las alteraciones iónicas paralelas a la acidosis, pero no se puede afirmar concluyentemente que no tenga alguna relación con la síntesis de la hormona antiraquítica.

Insuficiencia Renal.- Howard y colaboradores (9) han demostrado que el suero de pacientes con insuficiencia renal crónica inhibe in vitro la calcificación de cartílagos raquíticos mientras que el suero de sujetos normales o con otras formas de osteomalasia, no tiene este efecto. La retención de pirofosfatos que inhiben la mineralición podría ser una de las causas de raquitismo en la insuficiencia renal aunque el fenómeno es más complejo (84). En esta enfermedad hay además acidosis y no sólo se presenta raquitismo sino también osteitis fibrosa quística y calcificaciones metastáticas. Ultimamente se ha ensayado la 1 - $25(\mathrm{OH})_{2} \mathrm{D}_{3}$ en pacientes con insuficiencia renal crónica (85) observándose que dosis cercanas a las fisiológicas lograron curar el raquitismo en un caso. Se ha demostrado también que la hormona antiraquítica corrige el metabolismo cálcico alterado de la insuficiencia renal avanzada (86).

Hipofosfatasia.- Es una enfermedad hereditaria, de forma autosómica recesiva que puede provocar raquitismo neonatal grave o formas leves con enanismo y pérdida precoz de los dientes (82). La disminución de la fosfatasa alcalina en plasma sirve para diferenciarla de las otras formas en que la fosfatasa está siempre aumentada. Según Fanconi la vitamina $\mathrm{D}$ en esta enfermedad está contraindicada porque puede favorecer el desarrollo de una nefrocalcinosis debido a la frecuente hipercalcemia (82). Su patogenia podría relacionarse con la falta de fosfatasa para anular el efecto inhibidor de los pirofosfatos.

En resumen, la etiopatogenia de las distintas formas clínicas de raquitismo, se podría simplificar diciendo que todas coinciden en provocar hipofosfemia con hiperfosfaturia excepto la hipofosfatasia que podría considerarse como cuadro clínico aparte. Esta simplificación, esquematizada en la Fig. 2, destaca la unidad patogénica de las distintas formas clínicas de raquitismo pero de ningún modo pretende ser completa. Particularmente la patogenia de las formas carenciales, se explica sólo en parte por el hiperparatiroidismo secundario que les es común. Queda todavía por aclarar la posibilidad de alguna otra función directa de la hormona antiraquítica sobre la mineralización de la matriz ósea, u otras acciones que expliquen mejor por qué su carencia provoca las alteraciones óseas raquíticas que no son las mismas del hiperparatiroidismo.

\section{REFERENCIAS}

1.- Ham, A. W.: Tratado de Histología. Cap. 18. Hueso. pp. 388-459. 6 $6^{\text {a }}$ Ed. Interamericana. México, 1970.

2.- Theiler, K.: Citogénesis del tejido conjuntivo del cartilago, del hueso y de la musculatura. En: Opitz, H y Schmid, F. Enciclopedia pedátrica. Ed. Morata, Madrid, 1970. v. 6. pp. 24-29.

3.- Ramalingaswami, V. and Deo, M. G.: Experimental Protein-Calorie malnutrition in the rhesus monkey. In: Calorie Deficiencies and protein decificiencies. Proceedings of a Colloquium held in Cambridge April 1967. Little, Brown and Company, Boston. 1968. pp. 265-275.

4.- Thomson, A. H.: The later results in man of malnutrition in early life. In Calorie Deficiencies and protein deficiencies. Proccedings of a Colloquium held in Cambridge April 1967. Little, Brown and Company, Boston, 1968. pp. 289-299.

5.- Reifenstein, E. C. Jr.: Tratornos metabólicos del esqueleto. Consideraciones Generales. En: Harrison, T. R. y col.: Medicina Interna. 3a Ed. La Prensa Médica Mexicana, México, 1970, pp. $688-700$.

6.- Guyton, A.: Tratado de Fisiología Médica. 3: Ed. Interamericana, México, 1967, p. 1056.

7.- Litvak, J.: Diagnóstico de las Enfermedades de las glándulas paratiroides y del hueso. E. Andrés Bello, Santiago de Chile, 1968. 
8.- Fomon, S. J.: Infant. Nutrition. W. B. Sauders Company, Philadelphia, 1967, p. 26.

9.- Potts, J. T., Jr., Deftos, L. J.: Parathyroid Hormone, Thyrocalcitonin, Vitamin D, Bone and Bone Mineral Metabolism. In Duncan's Diseases of Metabolism. Endocrinology and Nutrition. W. B. Saunders Company. Philadelphia, 1969, pp. 9041082.

10.- Page, L. A.: Clinical Implication of Recent Advances in the understanding of calcium metabolism. Adv. Pediatr. 17: 317, 1970.

11.- Houssay, B. A. y col.: Fisiología Humana. Cap. 48 Metabolismo Mineral, pp. 580-606. Ed. El Ateneo. Buenos Aires, 1973.

12.- Bordier, Ph. J.: Aspectos histológicos del remodelamiento óseo. Triángulo. 12: 85-92,1973.

13.- Schnitman, S.: Osteopatias metabólicas. Ed. Stilcograf. Buenos Aires, 1968.

14.- Jowsey, J.: Valoración microradiográfica de la estructura del hueso. Triángulo. 12: 93-102, 1973.

15.- Reifenstein, E. C. Jr.: Trastornos Metabólicos deI esqueleto. Osteoporosis. En: Harrison, T. R. y col.: Medicina Interna $3^{\text {a }}$ Ed. La Prensa Médica Mexicana, México 1970, pp. $701-705$.

16.- Platt, B. S.: Experimental Protein-Calorie Deficiency. In Calorie Deficiencies and protein deficiencies. Proceedings of a Colloquium held in Cambridge. Abril 1967. Little. Brown and Company. Boston, 1968, pp. 237-248.

17.- Bergstrom, W. H., and Gardner, L. I.: Trastornos metabólicos con lesiones óseas. En Nelson, W. E., Vaughan, V. C. y McKay, J. R.: Tratado de Pediatría. Salvat Editores, S. A., Barcelona, 1973, pp. 1401-1411.

18.- Meneghello, J. y col.: Lecciones de Pediatría Clínica y Social. III. Raquitismo por carencia de vitamina D. Clínica y Tratamiento, pp. 57-62. Ed. Universitaria, Santiago, Chile 1966.

19.- Tanaka, Y. $y$ and De Luca, H. F.: Role of 1-25 Dihydroxyvitamin $D_{3}$. Proc. Nat. Acad. Sci. USA. 71: 1040-1044, 1974

20.- Royer, P.: Metabolismo del calcio. en Debré, R. y Lelong, M. Pediatría. Ed. JIMS. Barcelona, 1968, pp. 1615-1618.

21.- Rasmussen, H. and Reifenstein, C. Jr.: The Parathyroid Glands in Williams, R. H. Texbook of Endocrinology. Third Edition, W. B. Saunders Company. Philadelphia and London 1962, pp. 731879.

22.- NRC.: Recomendaciones Nutricionales Diarias, 1968. Departamento de Salud Pública y Medicina Social. Area Norte, Santiago, Chile 1971.

23.- Rasmussen, H.: Regulación hormonal de la función de la célula ósea. Triángulo, 12: 103-110, 1973.

24.- Harrison, Harold E., and Harrison, H. C.: Intestinal transport of phosphate: action of vitamin D, calcium and potasium. American Journal of Phisiology, 201: 1007-1012, 1961.
25.- Szloma, K. and Schachter, D.: Effects of Vitamin $\mathrm{D}$ on Phosphate transport and incorporation into mucosal constituents of rat intestinal mucosa. The journal of Biological Chemistry, 244: 211-217, 1969.

26.- Wasserman, R. H. and Taylor, A. N.: Intestinal Absortion of Phosphate in the chick: Effect of Vitamin $\mathrm{D}_{3}$ and other parameters. The Journal of Nutrition. 103: 586-599, 1973.

27.-- Schwarz, G.: Metabolismo del Calcio y del Fósforo. En Opitz, H. y Schmid, F.: Enciclopedia Pediátrica. Ed. Morata, Madrid. v. IV, pp. 347-362, 1967.

28.- Fraser, D. and Salter, R. B.: The diagnosis and management of the various types of rickets. Pediat. Clin. North. Am. 5: 417-441, 1958.

29.- De Luca, H. F.: Vitamin D 1973. The Amer. J. of Med. 57: 1-12, 1974.

30.- Kodicek, E.: The biosynthesis of ${ }^{14} \mathrm{C}$-labelled ergocalciferol. Biochem. J. XXV: 60-69, 1955.

31.- De Luca, H. F.: 25-hydroxicholecalciferol, the probable metabolically active form of vitamin $D$. Am. J. Clin. Nutr. 22: 412-424, 1969.

32.- Blunt, J. W., DeLuca, H. F. and Schnoes, H. K.: 25-Hydroxycholecalciferol. A biologically Active Metabolite of Vitamin $\mathrm{D}_{3}$. Biochemystry, 7 : 3317 $3322,1968$.

33.- Stamp, T. C. B.: Vitamin D metabolism. Recent advances. Arch. Dis. Child. 48: 2-7, 1973.

34.- Schachter, D. Finkelstein, J. and Kawarski, S.: Metabolism of vitamin D. I. Preparation of radioactive vitamin $\mathrm{D}$ and its intestinal absorption in the rat. J. Clin. Invest. 43: 787-796, 1964.

35.- Avioli, L. V.: Absorption and metabolism of vitamin $\mathrm{D}_{3}$ in man. Am. J. Clin. Nutr. 22: 437-446, 1969.

36.- Avioli, L. V., Lee, S. W., McDonald. J. E. Lund, $J$, and DeLuca, H. F.: Metabolism of vitamin $\mathrm{D}_{3}-{ }^{3} \mathrm{H}$ in human subjects: distribution in blood, bile, faeces, and ${ }^{3}$ urine. J. Clin. Invest. 46: 983992, 1967 .

37.- Fraser, D., Kooh, S. W., Kind, H. P., Holick, $M$. F., Tanaka, Y., and DeLuca, H. F.: Pathogene sis of Hereditary vitamin-D-Dependent Rickets. An Inborn Error of Vitamin D Metabolism Involving Defective Conversion of 25-Hydroxyvitamin $D$ to 1-alfa, 25 Dihydroxy vitamin. D New Engl. J. Med. 289: 817-822, 1973.

38.- Belsey, R., Clark, M. B., Bernat, M., Glowacki, J., Holick, M. F., DeLuca, H. F., and Potts, J. T.: The Physiologic Significance of Plasma Transport of Vitamin D and metabolites. Am. J. Med. 57: 28-33, 1974.

39.- Haddad, J. G., Stamp, T. C. B.: Circulating 25Hydroxyvitamin D in Man. Am. J. Med., 57: 57-62, 1974.

40.- Bhattacharya, M. H. and DeLuca, H. F.: The regulation of rat liver calciferol-25-hydroxilasa. J. Biol. Chem, 248: 2969-2974, 1973. 
41.- Holick, M. F., Schnoes, H. K., DeLuca, H. F.: Identification of 1-25 Dihydroxycholecalciferol a form. of vitamin $\mathrm{D}_{3}$ metabolically active in the intestine. Proc. Nat. Acad. Sci. USA. 68: 803-804, 1971.

42.- Rasmussen, H., Wong, M., Bikle, D and Goodman, D. P. B.: Hormonal Control of the Renal Conversion of 25-Hydroxycholecalciferol to 1-25-Dihydroxycholecalciferol. J. Clin. Invest. 51: 2502-2504, 1972 .

43.- Norman, A. W.: 1 - 25-Dihydroxyvitamin $\mathrm{D}_{2}: A$ Kidney Produced Steroid Hormone Essential to Calcium Homeostasis. The American Journal of Medicine. 57: 21-27, 1974

44.- DeLuca, H. F.: Nuevos conocimientos sobra el metabolismo de la vitamina D. Triángulo. 12: 111$118,1973$.

45.-- Suda, T., DeLuca, H. F., Schnoes, H. K., Tanaka, Y. and Holick, M. F.: 25.26 Dihydroxycholecalciferol, a metabolite of Vitamin D, with intestinal Calcium Transport Activity. Biochemystri, 9: 4776$4780,1970$.

46.- Soergel K. H., Mueller, K. H., Gustke, R. F. and Geenen, J. E.: Jejunal Calcium Transport in Health and Metabolic Bone Disease: Effect of Vitamin D. Gastroenterology. 67: 28-34, 1974.

47.- Wasserman, R. H. y Taylor, A. N.: Significación fisiológica de la proteína fijadora del calcio dependiente de la vitamina D. Triángulo. 12: 119$127,1973$.

48.- Wasserman, R. H. and Taylor, A. N.: Vitamin $\mathrm{D}_{3}$ Induced Calcium Binding Protein in Chick Intestinal Mucosa. Science. 152: 791-793, 1966.

49.- Drescher, D and DeLuca, H. F.: Posible precursor of vitamin D Stimulated Calcium Binding Protein in Rats. Biochemistry. 10: 2308 - 2312, 1971.

50.- Hurwitz, S, and Bar, A.: Site of Vitamin D action in chick intestinc. Am. J. of Physiology. 222: 761-767, 1972.

51.- Suda, T., DeLuca, H. F., Schnoes, H. K. Ponchon, G., Tanaka, Y., and Holick, M. F.: 21-25 Dihydroxycholecalciferol. A metabolite of Vitamin $\mathrm{D}_{3}$ Preferentially active on Bone. Biochemistry. 9: 2917-2922, 1970.

52.- Raisz, L. G., Trummel, C. L., Holick, M. F. and DeLuca, H. F.: 1-25 Dihydroxycholecalciferol: A Potent Stimulator of Bone Resorption in Tissue Culture. Science. 175: 768-769, 1972.

53.- Puschett, J. B., Moranz, J. and Kurnick, W. S.: Evidence for a direct action of cholecalciferol and 25-hydroxycholecalciferol in the renal transport of phosphate, sodium and calcium. The J. Clin. Invest. 51: 373-385, 1972.

54.- Pechet, M. M. and Hesse, R. H.: Metabolic and Clinical Effects of Pure Crystaline 1 alfa-Hydroxyvitamin $\mathrm{D}_{3}$ and 1 alfa 25-Dihydroxy-vitamin $\mathrm{D}_{3}$ Studies of Intestinal Calcium Transport, Renal Tubular Function and Bone Metabolism. Am. J. Med. 57: 13-20, 1974.
55.- Bordier, Ph. J.: Aspectos histológicos del remodelamiento ósso. Triángulo. 12: 85-92, 1973.

56.- Balsan, S.: Vitamina D. Acquisitions recents. Pediatría XVI. Actas del XIV Congreso Internacional de Pediatría. 3 al 9 de octubre 1974. Buenos Aires. Ed. Médica Panamericana, 1974. t. 10 pp. $94-102$.

57.-- Peacock, M., Gallagher, J. C., and Nordin, B. E. C.: Action of 1 alfa-Hydroxyvitamin $\mathrm{D}_{3}$ on Calcium Absortion and Bone rasorption in Man. Lancet. 1: 385-5738, 1974.

58.- Hallick R. B. and DeLuca, H. P.: 25-Hydroxydihydrotachysterol. Biosynthesis in vivo and in vitro. The Journal of Biological Chemystri. 246: 5733-5738, 1971.

59.- Nassim. J. R., Saville, P. D., Cook, P. B. and Mulligan, L.: The effects of vitamin $\mathrm{D}$ and gluten - free die $t$ in idiopathic steatorrea. Quarterly Journal of M dicine. New Series XXVIII: 141162, 1959.

60.- Hahn, T. J., Birge, S. J., Scharp, C. R. and Avioli, L. V.: Phenobarbital-Induced Alterations in vitamin D Metabolism. J. Clin. Invest. 51: 741-748, 1972.

61.- Chesney, R. W. and Harrison, H. E.: Fanconi syndrome following bowel surgery and hepatitis reversed by 25 hydroxycholecalciferol. J. Pediat. 86: 857-861, 1975.

62.-Dint, C. E., Richens, A., Rowe, D. J. F., and Stamp T. C. B.: Osteomalasia with Long-term Anticonvulsant Therapy in Epilepsy. Br. Med. J. 4: 69-72, 1970.

63.-- Richens, A., and Rowe, D. J. F.: Disturbance of Calcium Metabolism by Anticonvulsant Drugs. Br. Med. J. 4: 73-76, 1970.

64.- Stamp, T. C. B.: Effects of Long term Anticonvulsant therapy on Calcium and vitamin D Metabolism. Proc. R. Soc. Med. 67: 64-68,, 1974.

65.- Medlinsky, H. L.: Rickets Associated with anticonvulsant medication Pediatrics, 53: 91-95, 1974.

66.- Latorre, H., Kenny F.: High Dosage Intravenous calcium therapy with hypomovilization. Pediatrics, 53: 100-104, 1974.

67.- Maciaran, N. and Lifshiz, F.: Vitamin D dependency rickets in institunalized, mentaly retarded children on long term anticonvusante therapy. II The response to 25 hydroxycolecalciferol and to vitamin D, Pediatr. Res. 7: 914 - 922, 1973.

68.- Liakakos, D., Papadopoulos, Z., Vlachos, P., Boviats, E., and Dionissios, O.: Serum alkaline phosphatase and urinary hydroxiproline values in children receiving phenobarbital with and without vitamin D. The Journal of Pediatries 87: 291296, 1975.

69.- Pitts, R. F.: Phisiology of the kidney and Body Fluids. Chapter 14, Renal Function in Renal Disease, pp. 270-284. Third Ed. Year Book Med. Pub. Inc. Chicago, 1974. 
70.- Staum, B. B., Hamburger, R. J. and Goldberg, M.: Tracer microinjection Study of $\mathrm{R} \approx$ nal Tubular Phosphate Reabsortion in the Rat. J. Clin. Invest. 51: 2271-2276, 1972.

71.- Matsuda, L., Takekoshi, Y. Shida, N., Fujieda, K.: Nagai, B., Arashima, S. Anakura, M. and $O k a$, Y.: Renal Tubular Acidosis and Skeletal Demineralization in patients on long term anti-convulsant theraphy. The Journal of Pediatrics, 87: 202-205, 1975.

72.- Guignerd, J. P. and Torrado, A.: Proximal renal tubular acidosis in Vitamin D. D-ficiency Rickets. Acta Pediatr. Scand. 62: 543-546, 1973.

73.- Fraser D., Kooh, S. W. Scriver, C. R.: Hyperparathyroidism as the cause of Hyperaminoaciduria and phosphaturia in human vitamin D deficiency. Pediat. Res. 1: 425-431, 1967.

74.- Torres-Goitia, J. Ochoa. E., Fernández, E., Vallejos, E., Radrigán, M. E.: Raquitismo refractario. Revista Chilena de Pediatría. Publicación en este ejemplar.

75.-Haussler, M. R., Zerwelh, J. E., Hesse, R. H. Rizzardo, E., Pechet M. M.: Biological activity of 1 alfa hydroxycholecalciferol, a synthetic ana$\log$ of the hormonal form of vitamin $\mathrm{D}_{3}$. Proc. Natl. Acad. Sci. USA. 70: 2248-2253, 1973.

76.- Sriver, Ch. R.: Rickets and the Pathogenesis of impaired tubular transport of phosphate and other solutes. Amer. J. Med. 57: 43-49, 1974.

77.- Prader, A.: Pseudo Vitamin D. Deficiency (Vitamino D Dependency) Pediatría XIX. Actas del XIV Congreso Internacional de Pediatría. 3-9 de octubre de 1974. Buenos Aires. Ed. Médica Panamericana, 1974 , t. 10 , p. 106 .
78.- Stern, C.: Principles of Human Genetics. 2a Ed. W. H. Freeman and Company. San Francisco and London, 1960, p. 234.

79.- Scriver, C. R., Glorieux, F. H., Reade, T. and T:nenhouse, H.: Familial hyphosphatemic (Xlinked) Rickets. Pediatría XIV. Actas del XVI Congreso Internacional de Pediatría, 3-9 de octubre de 1974. Buenos Aires. Ed. Médica Panamericana, 1974. t. 10, pp. 103-05.

80.- Scriver, C. R.: Familial Hypophosphatemia: The dilema of treatment. New Engl. J. Med. 289: 531$532,1973$.

81.-- Crawford, J. D.: Oncogenous Rickets. Pediatría XIV. Ac:as del XIV Congreso Internacional de Pediatría 3-9 de octubre de 1974. Buenos Aires, Ed. Médica Panamericana, 1974. t. 10, pp. 107-108.

82.- Fanconi, G.: Osteopatías de origen genético. Triángulo. 12: 129-138, 1973.

83.-- Ochoa, E. Borie, G., Cortés, M.: Acidosis tubular renal. Presentación de 2 casos clinicos. Revista Chilena de Pediatría 46: 111-114, 1975.

84.- Lumb, G. A., Mawer. E. B. and Stanbury, S. W.: The Apparent Vitamin D. Resistance of chronic Renal Failure. Am. J. Med. 50: 421-441, 1971.

85.- Henderson, R. G., Russell, R. G. G., Smith, R., Oliver, D. O., Walton, R. J., Small, D. G., Preston, C., Warner, G. T. and Norman, A. W.: Effects of 1-25-Dihydroxycholecalciferol on calcium absortion, muscle weakness, and bone disease in Chronic Renal Failure. Lancet. 1: 379-384, 1974.

86.- Brickman, A. S., Coburn, J. W., Norman, A. W., Massry, S. G.: Short-Term Effects of 1-25- Dihydroxycholecalferol on Disordered Calcium Metabolism of Renal Failure. An. J. Med. 57: 28-33, 1974. 